in the Annals of Surgory, vol. xxx. He has collected all the American cases of actinomycosis, sixty-five in number, reported to January, 1899. His statement that "the thoracic cases do badly, as a rule, no matter what treatment is followed," is in accordance with the prerailing opinion of the profession.

\section{TROPICAL ABSCESS OF THE LIVER.}

\section{E. F. ROBINSON, M.D.}

\section{KANSAS CITY, MO.}

With the continuous residence of our army in the tropics and the constant return to the United States of scores of invalided soldiers, the subject of tropical diseases has assumed new importance to the medical profession of this country. Probably no subject has been brought so intimately to its attention as that of abscess of the liver. Its relation to dysentery is known. and the frequent development of liver abscess late in the course of this disease explains its appearance so often in the United States.

From the earliest times dysentery has been the scourge of armies, but in our own campaign in the Philippines we have had superimposed on the malevolent influences of the massing of great bodies of men together the presence of an endemic disease. The record of the First Reserve Hospital in Manila for one year-March 1, 1899-1900 - shows that dysentery and diarrhea were the cause of more than one-fifth of the sickness in our army. In this year there were treated at this hospital alone 2251 cases of diarrhea and 1391 of pronounced dysentery. Dysentery and diarrhea followed directly the course of the rainy season. During June, July and August the sick report was crowded with these cases. Often whole companies would be stricken as with an epidemic. The sudden chilling and exposure of the first rains made the number of cases during the month of June more than three times as great as that of any other month. With the American in the tropics dysentery is always present, and with it abscess of the liver. The conditions which favor the development of the one makes more evident the existence of the other.

Numerous statistics demonstrate this fact. In 3680 dysentery autopsies made in various tropical countries and reported by Manson, 21 per cent. showed abscess of the liver. The exact number of cases among our own troops can not be definitely stated, but the prevalence of the disease and its intimate relation to amebic dysentery is well shown by the examination of the records of ninety-six dysentery autopsies performed at the First Reserve Hospital, Manila, during the year 1899. Abscess of the liver was present in twelve cases-over 12 per cent. While this number is a small one on which to base a statistical opinion, yet it gives very nearly the correct estimate of the percentage of liver abscesses developing in cases of chronic tropical dysentery among Europeans. The native population in the Philippines rarely develops this disease, although dysentery is common among them. Not a single case of liver abscess was seen among the natives in my own experience in the islands, and I am informed by well-educated Spanish and Philippino physicians that to them the disease is almost unknown.

The life led by Europeans in the tropics doubtless explains the frequency of liver abscess. Overeating and overdrinking, together with lack of exercise, favor hepatic engorgement, and the sudden chilling and exposure incident to the first few months of residence fur- nishes the exciting cause in the development of an acute amebie dysentery.

Although dysentery is without doubt a cause of tropical abscess, the part played by the ameba coli in its development is as yet undetermined. Whether it was a cause or a result in our own cases we have no evidence to offer. All were of the amebic type of dysentery. The organism was either found in the stools or the pathologic picture was so typical that search was not made for it. From the pus of the abscess, or the abscess wall itself, there is record of the ameba in only five cases. This should not, however, lead to the conclusion that the organism had not been present in the remainder, for most of these cases were long standing, with great destruction of liver substance, and a sufficiently persistent search was not made in every one. Only one case of liver abscess was not dysenteric, but was apparently a multiple idiopathic abscess. There was no history of dysentery or diarrhea, nor any postmortem evidence of previous amebic infection, and the organism could not be obtained from the liver pus.

Of the 13 cases on our records, 5 were single and 8 were multiple abscesses. As Manson ${ }^{1}$ very aptly remarks: "Whether the resulting abscess be single or multiple is more or less a matter of accident. If the weakened liver is efficiently inoculated at one point only, there will be only one abscess; if at many points, then there is multiple abscess."

The right lobe was most commonly affected. In only 2 instances was the left involved. Five cases of abscess of the liver came under our observation clinically; 3 were multiple abscesses in which nearly the whole liver substance was destroyed; 2 were large single abscesses. All 5 were operated on, and only 1 recovered.

In the diagnois of abscesses of the liver symptoms are of but little value. Local signs and the detection of pus by aspiration establishes the diagnosis. Great emaciation and an anemic jaundice are apparently commensurate with the destruction of liver substance. The word anemic is applied to this jaundice to indicate a peculiar "diluted," faded, almost dusky, yellow color of the skin common in these cases. A hectic temperature, a rapid running pulse, with the early development of typhoid symptoms are in proportion to the amount of pus present; for there seems to be no tendency to limit absorption in abscess of the liver. Sordes soon collects on the teeth and lips, and mental hebetude develops early. The pulse is rapid and running. often being out of all proportion to the temperature. It may remain at 140 to 160 , or even higher, for weeks just before death. Constipation is the rule, with offensive gray-colored stools, but diarrhea is common late in the disease. A leucocytosis is generally present, but is of little value in the diagnosis as it is very often assoeiated with localized peritonitis, or involvement of the mesenteric glands, in cases of chronic dysentery. Often the local and general symptoms of abscess may develop from a patch of necrosis without the formation of pus. In these cases it is evident aspiration would fail to detect the infected area in the early course of the disease. Two cases came to autopsy from dysentery, and the abscesses would have undoubtedly developed had life been prolonged. A localized peritonitis is present in most cases of long duration. Involvement of the diaphragm is evidenced by persistent cough with increased pain on deep inspiration. Rupture of the abscess into the pleura, with involvement of the lung, is the commonest form of spontaneous evacuation; two of our autopsies showed

1. Tropical Diseases, p. 364. 
this condition, and it was present in one operative case.

The great increase in the area of liver dulness, not only above and below, but particularly to the left of the median line was found in all of our cases. Persistent pain over the whole liver region, with a point of local tenderness just below the margin of the ribs in the anterior axillary line is present in most cases. Local bulging, increase in the width of the interspaces, with local edema of the right side makes abscess most probable, but aspiration alone establishes the diagnosis. In our cases a long needle of goodly size was used, so that the thickened pus might be drawn through it. A general anesthetic was given and the needle introduced in the mid-axillary line in the eighth interspace and passed in five or six different directions to its full extent. If this failed to detect pus additional punctures were made both in the right and left lobes, for very often a liver abscess may not be detected by the ordinary puncture in the eighth interspace. This condition was present in one of our cases, and it was not until additional punctures had been made near the median line below the ribs that an abscess was discovered in the left lobe.

In order to determine the extent of liver accessible to aspiration from this point a series of liver punctures were made with long needles, postmortem. The needles were left in situ and dissection carried down through the liver substance, when it was found that not onehalf of the organ was accessible from the eighth interspace. In one case an echinococcic cyst the size of a small orange lay in the posterior inferior portion of the right lobe toward the median line, and entirely escaped detection.

From these facts we were led to the conclusion that additional punctures other than in the eighth interspace must be made before an abscess of the liver can be excluded in the diagnosis. With ordinary care there is little danger of injuring the gall-bladder, or the large vessels, and with strict asepsis the operation is entirely justifiable as a means of differential diagnosis. Not an untoward complication resulted in twenty-one cases of aspiration. The presence of pus once established makes operation imperative. Only a limited number of patients recover; not from the fact that the operation is difficult, but because the abscess is generally multiple, or, if single, involves a great amount of liver substance, and comes to the surgeon only late in the course of the disease. Unless there is evidence of pointing, it is best to excise a portion of a rib and drain from the side, as there is thus less danger of infection and greater facility in gaining access to the abscess cavity.

Even in most extensive liver abscesses the lower anterior border of the liver is often not involved, the infectious process being confined to the substance of the organ. In many instances the abscess can be reached only with the greatest difficulty by the incision below the costal margin. By excising a portion of the eighth or ninth rib in the mid-axillary line there is almost no danger of infection, but little shock and the freest possible drainage in the most dependent position. Large double drainage tubes are used. The removal of a portion of a rib not only gives more room for exploration, but insures against compression of the tubes as the case progresses. If the liver is adherent, and the peritoneum or pleura thus protected by adhesions, the abscess may be opened at once. If there is danger of infecting either, a delay of forty-eight hours is obviously wise. The instruction to "stitch the capsule of the liver to the margin of the wound" is more didactic than practical. Rarely, if ever, can this be done, as the liver capsule- particularly that of an inflamed liver-is so friable that no stitches will ho]d, and but little protection would be afforded if they did. Simply packing the wound with iodoform gauze and waiting forty-eight hours will accomplish the desired result. On account of free hemorrhage in opening a deep-seated abscess the thermocautery may be used. but this is rarely necessary, as access can be readily gained to the abscess cavity by puncture with the finger or a blunt instrument. Severe hemorrhage, if it occurs, can be controlled by packing. Usually it is best simply to drain the abscess at operation and to use no irrigation for forty-eight hours, owing to the weakened condition of the patient and the danger of infecting the pleura or peritoneum. Normal salt solution, sterile water or weak antiseptic solutions should be used, as absorption is very great and strong antiseptics are dangerous. Under daily irrigation with such solutions the discharge will completely disappear in a surprisingly short time - a week or ten days even for a large abscess. Free stimulation and most nourishing foods are particularly essential in the after-treatment.

Case 10,635.-April 20, 1900, Private T. S., Co. F, 42d U. S. V., was seen. He had never been sick since childhood until six weeks before, when he was attacked with diarrhea. The symptoms of dysentery rapidly developed, and on his admission, April 25 , he was passing from five to twelve bloody mucous stools a day. His temperature was 101 , tongue dry, brown and cracked, pulse 140 and thready. The spleen was slightly enlarged, and the abdomen tympanitic and tender on pressure. A point of great tenderness was evident just below the ribs over the region of the gall-bladder. The liver extended from the fourth interspace to the level of the umbilicus, $1 \frac{1}{2}$ inches below the costal margin in the right mammillary line, and about three inches to the left of the left median line. He was in a condition of mental hebetude, and complained of little pain except at bowel movements. The blood count revealed a leucocytosis of 18,000 . Widal's reaction was negative and the plasmodium malariæ was not found. Under ether, an aspirating needle was inserted through the eighth interspace and pus was found on the first puncture. An incision $1 \frac{1}{2}$ inches long was made over the eighth rib, 1 inch of the rib excised and the abscess at once opened and drained. More than one pint of fetid liver pus escaped. The abscess cavity was not irrigated until the second day, and then daily irriga tions of sterile water were begun. The pus had completely dis appeared on the tenth day and the man was out of bed. Re covery was complete. Amebæ were found in the abscess cavity.

Case 8215.-Sergeant J. D., Troop E, 11th Cav., for many months had had chronic dysentery. On admission, Nov, 21 1899 , he was having from four to ten stools daily, his temperature was hectic, 99 to 102 , pulse 138 and weak, and he was very weak and greatly emaciated. For several weeks he had had marked tenderness over the region of the gall-bladder: this had greatly increased. Intermittent hiccough and a persistent dry cough were troublesome, and suggested involve ment of the diaphragm. The liver extended 1 inch below the costal margin in the nipple line, and there was marked bulging at this point and marked edema of the whole right side.

No aspiration was made in this case, as pus was evidenteither an abscess or a suppurating gall-bladder. An incision was made one inch below the ribs in the nipple line, down to the peritoneum. This was found adherent, and so the abscess, very large, "big as your head," was at once evacuated. The cavity was irrigated with normal salt solution and three large drainage-tubes inserted. The pulse was so weak during operation that three pints of normal salt solution were given intra venously and one pint by hypodermoclysis. Despite this and the freest stimulation, the patient steadily grew worse, and died five days later. The autopsy showed multiple amebic ab. scesses, which had destroyed nearly the whole liver substance.

Case 4786.-Private Wm. H. H.. Co. K. 13th U. S. Inf.. had never been ill until Aug. 4, 1899, when he developed a severe case of acute dysentery. From the onset his temperature was 
unusually high-103 to 105 . The discharges were of the characteristic mucus and blood, and so frequent that he was constantly on the bed-pan-more than forty movements in twenty-four hours. Almost from the first he complained of pain over the region of the liver. The organ rapidly enlarged, and by the twelfth day the whole right side was edematous. Aspiration revealed the presence of pus and incision was made in the mid-axillary line, excising a portion of the eighth rib. Only a small amount of pus was evacuated. The patient never rallied from the operation, and died on the thirteenth day. The autopsy showed one small abscess with several necrotic patches throughout the substance of the organ, which would undoubtedly have formed additional abscesses had the patient lived.

Case 10,741.-G. M. W., a clerk, was seen March $\tau, 1900$ There was no history of dysentery or diarrhea. The patient had been in the Orient five months, and in fair health until about four weeks before, when he rapidly lost weight without assignable cause. On admission to the hospital, March 7 , he was much emaciated and of a peculiar dusky, jaundiced color. His temperature was subnormal and his pulse rapid and feeble. Pain over the liver was constant, with the point of greatest tenderness one inch below the costal margin in the mammillary line. The organ was very much enlarged, extending four finger breadths below the ribs. Under ether the aspirating-needle revealed pus. An incision 21/2 inches long was made below the costal border in the right nipple line. The liver was found adherent to the parietal peritoneum. On attempting to open the abscess such free hemorrhage occurred that the wound was packed with iodoform gauze and partially closed with silkworm gut sutures. Further operative procedure was delayed forty-eight hours, when it was intended to open the abscess with the Paquelin cautery. However, on separating the capsule from the diaphragm, about one pint of pus was evacuated. A drainage of double tubing was instituted, and the patient left the table in a very weak condition and died fortyeight hours later. The autopsy showed multiple abscesses of the liver involving nearly its whole structure. They varied in size from one to five inches in diameter and contained peculiarly fetid pus. There was no previous history or evidence of dysentery, nor could the ameba be found. Apparently the case was one of multiple liver abscess following general hepatitis. The mesenteric glands were much enlarged and the spleen was septic.

Case 220.-Private C. F. B., Co. 1, 4th Ci.S.Inf., gave a history of chronic dysentery of several months standing, but on the date of admission considered himself cured of that disease. His present illness began so insidiously that he could not state the time of its onset. During the preceding month he had lost greatly in weight and suffered from a constant steady pain in the epigastrium. On admission, the temperature was subnormal and his pulse rapid and feeble, with a dry brown tongue and mental hebetude, and his skin a dusky jaundiced color. The liver was much enlarged, extending fully three inches to the left of the median line and $11 / 2$ inches below the ribs. Aspiration at the eighth interspace, in five or six directions, failed to detect pus, so the needle was introduced into the left lobe from a point just to the right of the median line, $1 / 2$ inch below the rostal margin. Here puncture was success. ful and an incision was made down to the liver. The capsule and parietal peritoneum were adherent and a large abscess was opened and drained, with no irrigation. The patient rallied from the operation, but died on the fifth day. The autopsy revealed a large single abscess occupying the whole left lobe. It had perforated the diaphragm, and opening up the pleura had set up a septic pneumonia. Healed amebic ulcers were found in the intestines, but the ameba coli could not be distinguished.

Migration of Needles. - The Medical Press and Circular for April 24 reports the removal of about sixty needles from a domestic, aged 16. who says that five years ago, on a wager, she swallowed four or five packages. No inconvenience was noticed until recently, when the needles presented, usually by the head, in various portions of the body.

\section{THE PREVENTION OF INSANITY.}

\section{DANIFI, R. BR̃OWER, M.D., LL.D.}

Professor of Nervous and Mental Diseases, Rush Medical College; Professor of Diseases of the Nervous System and Clinical Medicine, Northwestern University Woman's Medical School, Etc. cHICAGO.

The necessity for more radical measures for the prevention of insanity must be manifest to any one who will judicially consider the question of its rapid increase; for while there has been some attempt to minimize this important question, yet it is capable of easy demonstration that insanity has increased out of all due proportion to the increase in population in the last thirty or forty years. The statistics of Great Britain show that the proportion of insane to the whole population in 1860 was 1 to 523 , and in 1890,1 to 320 ; and in Illinois the proportion of insane to the population is about 1 to 400 .

Preventive measures must come from the States. The learned and self-sacrificing profession to which we bzlong can show the way, but the state must compel people to walk therein. The state has assumed the care and maintenance of the insane; indeed, has made it a criminal offense for any one to restrain them without the consent of her courts. Inasmuch as the state has assumed this great responsibility, it is her duty, in the interests of altruism and of economy, to use every possible means for the prevention of insanity; to find out how this may be done; to educate the people as to methods of doing it and to enforce those methods. The state should, through her State Board of Health, furnish the people with information that they can comprehend as to the causes of insanity, and with methods that may be applied for its prevention; and the state should be as ready, with the same paternal power she uses in smallpox, cholera, etc., to enforce those methods that have the approval of the medical profession. The state should make her insane hospitals schools for the instruction of medical students in insanity. The state should, by competent authority, select by competitive examination senior students in medicine from our medical colleges, and place them in the hospitals for the insane as medical internes. At least half a dozen can be placed in each hospital for the insane in this state. These internes should remain at least one year, receiving board, lodging, and a small gratuity at the end of the service, and by this means at least thirty physicians every year, with mental attainments of a high order, will be sent forth with a good clinical knowledge of insanity, its prevention and its treatment, and become so many prophylactic centers in as many communities. But a few years would be necessary to have, from one end of Illinois to the other, an abundance of well-skilled medical men competent to solve this great problem.

The state should assist in providing treatment for carefully selected cases in wards connected with the. hospitals now to be found in every city and almost every town in the state. Admission to these wards should be by the same rules as apply to any other disease. The family of the patient would make but little objection to the prompt treatment of the case in such a manner, while it would decidedly object to the patient being sent to a hospital for the insane by a jury trial at some distant point, and especially to-day when our hospitals have become political machines for the reward of political favors. The patients in these hospital wards, under such scientific treatment as they may promptly obtain, will recover in very much larger proportion than is 